\title{
Risk Factors for Virus-induced Acute Respiratory Tract Infections in Children Younger Than 3 Years and Recurrent Wheezing at 36 Months Follow-Up After Discharge
}

\author{
Ambra Nicolai, MD, * Antonella Frassanito, MD, * Raffaella Nenna, MD, PhD, * Giulia Cangiano, MD, * \\ Laura Petrarca, MD, * Paola Papoff, MD, PhD, * Alessandra Pierangeli, MD, PhD, † Carolina Scagnolari, MD, PhD, $†$ \\ Corrado Moretti, $M D$, * and Fabio Midulla, $M D, P h D^{*}$
}

\begin{abstract}
Background: We sought to know more about how 14 common respiratory viruses manifest clinically, and to identify risk factors for specific virusinduced acute respiratory tract infections (ARTIs) in children younger than 3 years old and for wheezing at 36-month follow-up.

Methods: We retrospectively studied the clinical records for 273 full-term children (median age, 2.9 months; range, 0.26-39; boys, 61.2\%) hospitalized for ARTIs, whose nasopharyngeal specimen tested positive for a respiratory virus and 101 children with no history of respiratory diseases (median age, 8 months; range, 0.5-36.5; boys, 58.4\%). At 12, 24 and 36 months after children's discharge, all parents were interviewed by telephone with a structured questionnaire on wheezing episodes.

Results: The most frequently detected viruses were respiratory syncytial virus in bronchiolitis, human rhinovirus in pneumonia and human bocavirus in wheezing. Multivariate analysis identified, as risk factors for virusinduced ARTIs, the presence of siblings [odds ratio (OR): 3.0 (95\% confidence interval [CI]: 1.8-5.2)], smoking cohabitants (OR: $2.3(95 \% \mathrm{CI}$ : 2-4.2)] and breastfeeding lasting less than 3 months [OR: 0.5 (95\% CI: $0.3-0.9)]$. The major risk factor for respiratory syncytial virus-induced ARTIs was exposure to tobacco smoke [OR: 1.8 (95\% CI: 1.1-3.2)]. Risk factors for human rhinovirus-induced ARTIs were attending day-care [OR: 5.0 (95\% CI: 2.3-10.6)] and high eosinophil blood counts [OR: 2.6 (95\% CI: $1.2-5.7)]$. The leading risk factor for recurrent wheezing was exposure to tobacco smoke [OR: 2.5 (95\% CI: 1.1-15.6)].

Conclusions: Each respiratory virus leads to a specific clinical manifestation. Avoiding exposing children to tobacco smoke might restrict viral spread from sick parents and siblings to younger children, prevent severe respiratory diseases, and possibly limit sequelae.
\end{abstract}

Key Words: acute respiratory tract infections, respiratory viruses, risk factors, wheezing

(Pediatr Infect Dis J 2017;36:179-183)

A cute virus-induced respiratory tract infections (ARTIs) manifest clinically in various ways, depending on host age and pathogen virulence and can affect the entire respiratory tract from the nose to the lungs. ${ }^{1-8}$ ARTIs are the most frequent diseases in

Accepted for publication July 14, 2016.

From the *Department of Pediatrics and Infantile Neuropsychiatry, and †Department of Molecular Medicine, "Sapienza" University Rome, Rome, Italy.

A.N. and R.N. interpreted data for the work and drafted the work; A.F., G.C. and L.P. acquired the data; A.P. and C.S. performed and interpreted analysis; P.P. and C.M. revised the work critically for important intellectual content and F.M. conceived and designed the work.

The authors have no funding or conflicts of interest to disclose.

Address for correspondence: Fabio Midulla, MD, PhD, Department of Paediatrics, "Sapienza" University Rome, V.le Regina Elena 324, 00161, Rome, Italy. E-mail: midulla@uniroma1.it.

Copyright (C) 2016 Wolters Kluwer Health, Inc. All rights reserved.

ISSN: 0891-3668/17/3602-0179

DOI: $10.1097 /$ INF.0000000000001385 children and the most common reason for parents to consult a pediatrician. In the United States, the incidence of respiratory viral infections is 6.1-6.8 cases/yr in infants and 10.0 in children attending day-care. Overall, ARTI-related morbidity accounts for $30 \%-50 \%$ of work days lost for parents and $60 \%-80 \%$ of school days lost for children. ${ }^{1,9}$ Many respiratory viruses can cause ARTIs in children, and each virus follows a specific seasonal activity pattern. ${ }^{10,11}$

Studies investigating the various risk factors for virusinduced ARTIs have yielded contrasting results. Known risk factors for viral respiratory diseases in children are passive smoke and overcrowding, whereas the role of breastfeeding is controversial. ${ }^{2,12,13}$

Children with ARTIs, especially those infected with respiratory syncytial virus (RSV), are at risk for recurrent wheezing and asthma. ${ }^{8,14,15}$ Studies in the past 10 years link recurrent wheezing also to human rhinovirus (hRV) infection. ${ }^{16}$ Although ample evidence describes the incidence and clinical manifestations related to RSV and hRV, $, 7,8,16$ epidemiologic and clinical information about other respiratory viruses are limited and contrasting. ${ }^{14,17-20}$ Few studies have compared the clinical manifestations and risk factors for specific respiratory virus in children with ARTIs. ${ }^{1,10}$ Extending current epidemiologic knowledge would help in managing children with ARTIs and possibly in reducing the incidence of long-lasting respiratory sequelae. ${ }^{16}$

In this retrospective study, we sought more reliable clinical information on virus-induced ARTIs and on risk factors for recurrent wheezing. To do so, we reviewed the clinical records for children hospitalized with ARTIs during 5 annual epidemic seasons, whose nasopharyngeal specimen tested positive for a specific respiratory virus in a panel of 14 . For comparison, we analyzed data for children with no history of respiratory diseases attending the pediatric department over the same period. As the outcome measure to analyze possible risk factors for ARTIs, we compared demographic characteristics in the 2 groups. As the outcome measure to analyze risk factors for recurrent wheezing at 12, 24 and 36 months after hospitalization, we interviewed parents with a structured questionnaire designed to elicit information for comparing children with ARTIs who wheezed and those who did not.

\section{MATERIALS AND METHODS}

\section{Patients and Controls}

We retrospectively studied 273 full-term children (cases: median age, 2.9 months; age range, 0.26-39 months; boys, 61.2\%) hospitalized at the pediatric emergency department, "Sapienza" University Rome (Italy), from October 2009 to May 2014, except for June-August in each year. To be eligible, children had to be $0-3$ years old, hospitalized for an ARTI, and to have a nasopharyngeal specimen that tested positive for a respiratory virus. Nasopharyngeal specimens had been prospectively tested using a panel of specific reverse transcription-polymerase chain reaction 
assays detecting 14 respiratory viruses $[\mathrm{RSV}$, influenza virus A and $\mathrm{B}$, human coronavirus OC43, 229E, NL-63 and HUK1, adenovirus, hRV, parainfluenza virus $1-3$, human metapneumovirus (hMPV) and human bocavirus $(\mathrm{hBoV})]$ as reported in our previous papers. ${ }^{4,10}$ All polymerase chain reactions were optimized to detect at 10-100 input plasmid copies. ${ }^{10}$

Among the 273 patients enrolled, virus testing identified RSV in 148 children's specimens $(54.2 \%)$, hRV in $67(24.5 \%)$, hMPV in $13(4.8 \%), \mathrm{hBoV}$ in $10(3.7 \%)$ and 29 specimens $(10.6 \%)$ were coinfected $(\mathrm{RSV}+\mathrm{hRV}, \mathrm{RSV}+\mathrm{hBoV}, \mathrm{RSV}+\mathrm{hMPV}, \mathrm{RSV}+$ influenza, RSV + hRV + hBoV, RSV + hRV + hMPV, hRV + hBoV, $\mathrm{hRV}+\mathrm{hMPV}, \mathrm{hRV}+$ parainfluenza 3 and $\mathrm{hBoV}+$ parainfluenza 1$)$ and in 6 children $(2.2 \%)$ with other viruses (adenovirus, coronavirus and influenza).

For comparison, we enrolled 101 children, $0-3$ years old (median age, 8 months; age range, $0.5-36.5$; boys, 58.4\%), attending the pediatric outpatient clinic over the same period, with no virusrelated ARTIs and no history of respiratory diseases. To parents of children in both groups, we administered a structured questionnaire seeking demographic data. Studied variables included sex, age, history of breastfeeding, family history of asthma and atopy, presence of siblings, patient and siblings day-care attendance, family smoking habits, presence of atopic eczema and wheezing before admission. For breastfeeding history, we analyzed the length of breastfeeding, and ascertained whether breastfeeding lasted more than 3 months (or the entire lifetime if children were younger than 3 months).

For cases, on admission, we searched the clinical records for data on the following clinical and laboratory variables: presence of rhinitis, pharyngitis, otitis, vomiting, diarrhea and fever (temperature, $>37.5^{\circ} \mathrm{C}$ ). At admission, to each of the 273 patients, we assigned a clinical severity score ranging from 0 to 6 , assessing respiratory distress as previously described. ${ }^{8}$ Laboratory values recorded were blood hemoglobin, white blood cell count, platelets, serum glucose concentration, blood $C$-reactive protein concentration, serum glutamic oxaloacetic transaminase, serum glutamate pyruvate transaminase, serum gamma-glutamyl transpeptidase and serum sodium $\left(\mathrm{Na}^{+}\right)$.

All children's parents agreed and gave written informed consent to participate in the study, which was approved by the hospital institutional review board.

\section{Follow-up}

One of the authors (A.F.) interviewed patients' families using a standardized structured telephone questionnaire seeking information on the presence of wheezing episodes 12, 24 and 36 months after the child's discharge. Follow-up results were considered positive if children experienced more than 2 pediatriciandiagnosed wheezing episodes per year.

\section{Statistical Analysis}

Statistical data were analyzed with SPSS version 21.0 (SPSS Inc., Chicago, IL). Our descriptive analysis included percentages for discrete variables, median and range for continuous variables. Differences among groups of viruses were compared using a nonparametric test for median comparison (Mann-Whitney test). The $\chi^{2}$ test was applied to analyze categorical independent variables qualitatively. A multiple-way analysis of variance was run to test differences in virus groups. A multivariate regression analysis with odds ratios (ORs) and their corresponding 95\% confidence intervals (CIs) and including all statistically significant variables in a univariate analysis was run to identify risk factors for hRV- and RSV-induced ARTIs, and for recurrent wheezing during follow-up. In the multivariate analysis seeking risk factors for hRV and RSV, data were corrected by age. $P$ values $<0.05$ were considered to indicate statistical significance.

\section{RESULTS}

During the 5 annual epidemic seasons from 2009 to 20014 studied, virus distributions peaked between November and April, and most viruses were detected between December and January. RSV followed this distribution pattern, whereas hRV was almost equally distributed over the 5 years (Fig. 1A). In the 2011-2012 season, hRV infections increased, whereas RSV infections decreased (Fig. 1B). Virus distribution varied according to age: in younger children, 0-6 months old, the most frequent ARTIinduced viruses were RSV and hMPV, whereas in older children, 24-36 months of age, they were hRV and hBoV (Fig. 1C).

\section{Risk Factors for ARTIs}

Demographic data showed a higher though not significantly higher frequency of boys in the case group than in the control group (Table 1). Questionnaire answers indicated significantly shorter lasting and less frequent breastfeeding in children with ARTIs than in control children $\left(P<0.001\right.$ and $P=0.006$, by $\chi^{2}$ test). The questionnaire also showed that infants with ARTIs more frequently had siblings than controls $(P<0.001)$ and had a more frequent family history of atopy (parents' eczema $P<0.01$ and siblings' asthma $P=0.04$ ). Finally, case children had more though not significantly more smoking cohabitants than control children.

The multivariate analysis identified, as risk factors for ARTIs, the presence of siblings [OR: 3.0 (95\% CI: 1.8-5.2)], smoking cohabitants [OR: 2.3 (95\% CI: 1.2-4.2)] and breastfeeding lasting at least 3 months [OR: 0.5 (95\% CI: 0.3-0.9)].

\section{Demographic Characteristics and Clinical Expressions of the 14 Viruses Tested}

Of the 273 children hospitalized with ARTIs, 181 (66.3\%) had bronchiolitis, 56 (20.5\%) wheezing, 20 (7.3\%) pneumonia and $16(5.9 \%)$ upper respiratory tract infections (URTIs). Of the 181 children in whom virus-induced ARTIs manifested clinically with bronchiolitis, nasopharyngeal swabs from 118 (65.2\%) tested positive for RSV, from 29 (16.0\%) tested positive for hRV and 21 $(11.6 \%)$ were coinfected. Of the 56 children who wheezed, nasopharyngeal swabs from $23(41.1 \%)$ tested positive for RSV and $22(39.3 \%)$ positive for hRV. Nasal swabs from $50 \%$ of children who tested positive to $\mathrm{hBoV}$ came from children who wheezed. Of the 20 children in whom virus-induced ARTIs manifested clinically with pneumonia, nasopharyngeal swabs from 11 (55\%) tested positive for hRV and $5(25 \%)$ positive for RSV. URTIs were almost equally distributed among all viruses: nasal swabs tested positive for $\mathrm{hRV}$ in $5(31.3 \%)$, RSV and coinfections in $2(12.5 \%)$, hMPV and other viruses in $3(18.8 \%)$ and $\mathrm{hBoV}$ in $1(6.3 \%)$ (Table 2$)$

Children infected with RSV were younger than those infected with other viruses, whereas those with $\mathrm{hBoV}$ were the oldest $(P=0.013)$ (Table 3$)$. Patients with $\mathrm{hBoV}$ were more frequently breastfed, and attended day-care more frequently than other children $(P<0.001)$. The percentage of children with previous wheezing was higher in children infected with hRV than in those infected with $\operatorname{RSV}\left(P<0.001\right.$, by $\chi^{2}$ test $)$. Among clinical variables, the records described diffuse crackles in $90.5 \%$ of RSV-infected children, and wheezing in $70 \%$ of those infected with hBoV. No significant differences were found in clinical severity scores or laboratory data between the 14 viruses tested.

\section{Risk Factors for hRV and RSV ARTIs}

Multivariate regression analysis identified as a risk factor for RSV ARTIs exposure to tobacco smoke [OR: 1.8 (95\% CI: 1.1-3.2)] and as risk factors for hRV infections, attending day-care [OR: 5.0 (95\% CI: 2.3-10.6)] and eosinophil counts exceeding 300/mm $\mathrm{mm}^{3}$ [OR: 2.6 (95\% CI: $\left.1.2-5.7\right)$ ]. 


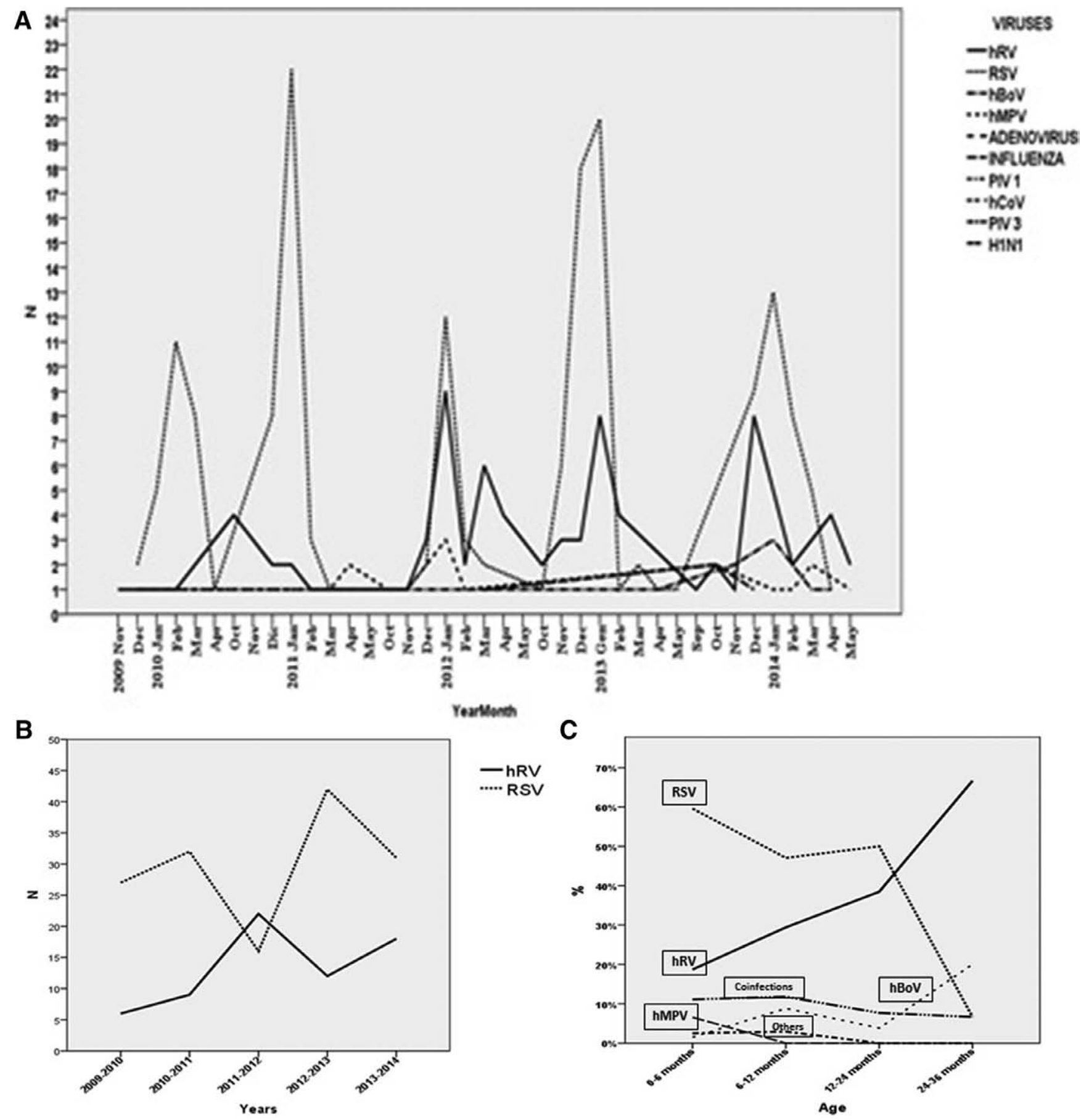

FIGURE 1. A: Virus distribution during the 5 annual epidemic seasons; B: human rhinovirus and respiratory syncytial virus distribution during 5 annual epidemic seasons and C: virus distribution in different age groups.

\section{Risk Factors for Recurrent Wheezing 12, 24 and 36 Months After Hospitalization for ARTIs}

At 12-month follow-up, the only risk factor multivariate regression analysis identified for recurrent wheezing was the presence of previous wheezing [OR: 4.9 (95\% CI: 1.9-12.2)]. Conversely, at later follow-up, the leading risk factor for recurrent wheezing was exposure to tobacco smoke [at 24 months, OR: 2.3
(95\% CI 1.2-4.5) and at 36 months, OR: 2.5 (95\% CI: 1.1-15.6)] regardless of clinical expression and the specific viral pathogens tested.

\section{DISCUSSION}

In this retrospective study investigating the clinical records for 273 children younger than 3 years old hospitalized for 
TABLE 1. Demographic Characteristics in Children With ARTIs and Control Children Without Respiratory Diseases

\begin{tabular}{lccc}
\hline \hline & $\begin{array}{c}\text { Children } \\
\text { With ARTIs } \\
(\mathrm{N}=273)\end{array}$ & $\begin{array}{c}\text { Control } \\
\text { Children } \\
(\mathrm{N}=101)\end{array}$ & $P$ \\
Variables & 61.2 & 58.4 & $\mathrm{NS}$ \\
\hline Boys, \% & $2.9(0.26-36)$ & $8.0(0.5-36)$ & $\mathrm{NS}$ \\
Median age in months (range) & $3.0(0-15)$ & $<0.001$ \\
Median breastfeeding months & $1.26(0-15)$ & & \\
$\quad$ (range) & 55.3 & 70.3 & 0.006 \\
Breastfeeding $>3$ mo, \% & & & \\
Parents with & 17.9 & 10.9 & $\mathrm{NS}$ \\
$\quad$ Asthma, \% & 9.2 & 2.0 & 0.01 \\
$\quad$ Eczema, \% & 13.6 & 5.9 & 0.04 \\
Siblings with & 8.0 & 8.9 & $\mathrm{NS}$ \\
$\quad$ Asthma, \% & 68.5 & 48.5 & $<0.001$ \\
$\quad$ Eczema, \% & 46.2 & 34.7 & 0.06 \\
Presence of siblings, \% & 12.1 & 15.6 & $\mathrm{NS}$ \\
Tobacco smoke exposure, \% & & & \\
Maternal smoking during & 4.8 & 6.9 & $\mathrm{NS}$ \\
$\quad$ pregnancy, \% & & & \\
Patient's eczema, \% & & &
\end{tabular}

for wheezing and pneumonia. The leading risk factor for recurrent wheezing developing at 36 months after hospital discharge was smoke exposure.

As well as extending current knowledge on viral pathogens in children with ARTIs, we confirmed the well-known winter epidemic peak for RSV and hMPV, whereas hRV maintained a steady epidemic curve throughout the year, reaching 2 small peaks in autumn and spring. ${ }^{4,17}$ The RSV decrease accompanied by a hRV increase during the 2011-2012 season might suggest an epidemiologic relationship between the 2 viruses.

A strong point in this study is that to analyze risk factors in children hospitalized for ARTIs, we tested children with a panel of 14 respiratory viruses. This experimental approach showed that the risk factors significantly influencing hospitalization for ARTIs are a family history for atopy, having siblings in the family, being exposed to passive smoke and not being breastfed, independently from the specific causative respiratory virus and its clinical expression. These observations support and extend also to other viruses previous similar findings from others who focused mainly on RSV infections..$^{2,412,13}$ Preventive measures designed to modify the identified risk factors and improving breastfeeding could reduce ARTIs thus substantially saving health expenditure.

TABLE 2. Viruses Identified According to the Different Clinical

Manifestations

\begin{tabular}{lccccc}
\hline \hline & $\begin{array}{c}\text { Bronchiolitis } \\
(\mathrm{N}=181)\end{array}$ & $\begin{array}{c}\text { Wheezing } \\
(\mathrm{N}=56)\end{array}$ & $\begin{array}{c}\text { Pneumonia } \\
(\mathrm{N}=20)\end{array}$ & $\begin{array}{c}\text { Upper Respiratory } \\
\text { Tract Infections } \\
(\mathrm{N}=16)\end{array}$ & $P$ \\
\hline Viruses & 118 & 23 & 5 & 2 & $<0.001$ \\
RSV $(\mathrm{N}=148)$ & 29 & 22 & 11 & 5 & \\
hRV $(\mathrm{N}=67)$ & 21 & 4 & 2 & 3 & \\
Coinfections $(\mathrm{N}=29)$ & 8 & 1 & 1 & 3 \\
hMPV $(\mathrm{N}=13)$ & 3 & 5 & 1 & 3 \\
hBoV $(\mathrm{N}=10)$ & 2 & 1 & 0 & \\
Other viruses $(\mathrm{N}=6)$ & & & & \\
\hline
\end{tabular}

Other viruses: influenza virus, $\mathrm{N}=4$; adenovirus, $\mathrm{N}=1$; coronavirus, $\mathrm{N}=1$. $P$ values by 4 -way analysis of variance.

TABLE 3. Clinical Characteristics Listed According the Viruses Identified in Children With Acute Respiratory Tract Infections

\begin{tabular}{|c|c|c|c|c|c|c|c|}
\hline Data & $\begin{array}{c}\mathrm{RSV} \\
(\mathrm{N}=148)\end{array}$ & $\begin{array}{c}\mathrm{hRV} \\
(\mathrm{N}=67)\end{array}$ & $\begin{array}{l}\text { Coinfections } \\
\quad(\mathrm{N}=29)\end{array}$ & $\begin{array}{l}\text { hMPV } \\
(\mathrm{N}=13)\end{array}$ & $\begin{array}{c}\mathrm{hBoV} \\
(\mathrm{N}=10)\end{array}$ & $\begin{array}{l}\text { Other Viruses } \\
\qquad(\mathrm{N}=6)\end{array}$ & $P$ \\
\hline Boys, $\%$ & 64.1 & 62.7 & 51.7 & 53.8 & 50.0 & 50.0 & NS \\
\hline $\begin{array}{l}\text { Breastfeeding in months } \\
\quad \text { (range) }\end{array}$ & $1.3(0-14.0)$ & $1.1(0-15.0)$ & $1.5(0-7.0)$ & $1(0-4.5)$ & $2(0-8.0)$ & $2.5(0.96-4.7)$ & NS \\
\hline Breastfeeding $>3 \mathrm{mo}, \%$ & 54.7 & 50.7 & 62.1 & 46.2 & 60.0 & 66.7 & NS \\
\hline Day-care, $\%$ & 8.1 & 31.3 & 10.3 & 7.7 & 40.0 & 0 & $<0.001$ \\
\hline Passive smoking, $\%$ & 50.7 & 44.8 & 31.0 & 30.8 & 50.0 & 50.0 & NS \\
\hline Previous wheezing, $\%$ & 9.5 & 31.3 & 20.7 & 7.7 & 30.0 & 16.7 & 0.003 \\
\hline Rales, $\%$ & 90.5 & 80.6 & 86.2 & 69.2 & 90.0 & 50.0 & $<0.02$ \\
\hline Wheeze, $\%$ & 33.1 & 37.3 & 24.1 & 30.7 & 70.0 & 0 & NS \\
\hline Severity score (range) & $3(0-8)$ & $3(0-7)$ & $3(0-6)$ & $2(0-3)$ & $2(0-6)$ & $1(0-4)$ & NS \\
\hline
\end{tabular}

$P$ values by multiple-way analysis of variance.

NS indicates not significant.

virus-induced ARTIs, we identified, as risk factors a family history of atopy, the presence of siblings, passive smoke and breastfeeding for less than 3 months. By testing a panel of 14 respiratory viruses, we identified RSV as the most common causative viruses in the children with ARTIs responsible mainly for bronchiolitis, and hRV
Accumulating evidence over the past 2 decades shows that several respiratory viruses can act as triggers for ARTIs. ${ }^{1-9}$ In our cohort of children $<3$ years of age, the most frequently detected viruses were RSV and hRV, followed by hMPV and hBoV. In a previous longitudinal study from our laboratory in children $0-14$ years 
of age hospitalized for ARTIs, we identified RSV as the main causative pathogen, followed by hRV and parainfluenza virus (PIV)-3. ${ }^{4}$ A similar study involving 404 children $0-16$ years of age showed that nasopharyngeal samples from $51 \%$ of patients hospitalized for lower respiratory tract infections tested positive for RSV and $28 \%$ for $\mathrm{hRV}, 5$ whereas in recent years, El Kholy et $\mathrm{al}^{7}$ found a $22.9 \%$ incidence of RSV in respiratory infections in children up to 14 years, hospitalized with an ARTI. Our results in children under 3 years fit in with previous findings. We presume that the high RSV in our study reflects the fact that our cohort of 273 children included $232(85.0 \%)$ infants younger than 1 year of age.

As well as confirming the incidence and clinical manifestations of RSV and hRV in children with ARTIs, ${ }^{3,17}$ our findings extend currently limited and contrasting epidemiologic and clinical knowledge on $\mathrm{hBoV}^{4,12,18,21}$ and hMPV in children. ${ }^{10,12,17,19,20}$ When we analyzed how the 14 respiratory viruses manifest clinically, the main diagnoses were bronchiolitis, wheezing and pneumonia. The virus most frequently causing bronchiolitis was RSV, whereas the causative agent in children with pneumonia was hRV. The viral pathogens most frequently causing wheezing were RSV (41.1\%) and hRV (39.3\%). A somewhat unexpected finding was that among 10 patients in whom nasopharyngeal swab testing identified $\mathrm{hBoV}, 5$ (50\%) wheezed. Nasopharyngeal swab testing identified hMPV mainly in children with bronchiolitis and URTIs, as in previous reports. ${ }^{10,12,17,19,20}$

Confirming ample evidence that respiratory virus testing commonly identifies more than 1 causative pathogen in patients with ARTIs, we found that $10.6 \%$ of children tested positive for 2 or more viruses. The most frequent coinfections were those with RSV and $\mathrm{hRV}$, and they most frequently manifested clinically with bronchiolitis. Others found a lower coinfection rate $(4.5 \%)$ in children with lower respiratory tract infections (LRTIs) 0-14 years of age: mainly RSV followed by adenovirus. ${ }^{7}$ Our results also confirmed that illnesses related to coinfections were no more severe than those related to single viruses. ${ }^{5,6,14}$

When we analyzed demographic features, clinical and laboratory data among viruses, we found no significant difference, except for age at admission. Patients with $\mathrm{hBoV}$ were older than those infected with RSV or other viruses, and more frequently attended day-care. In a similar study on children younger than 3 years with pneumonia, RSV prevalence decreased with increasing age, whereas hMPV and hRV remained steady at all ages. ${ }^{14}$ Conversely, a study conducted in children with LRTIs showed that patients with hMPV were older than infants with RSV. ${ }^{20}$

Few studies have focused on the possible risk factors for hRV ARTIs. In our study, we identified day-care attendance together with increased blood eosinophil numbers during hospitalization. These data could confirm the well-known association between this virus and subsequent development of allergy sensitization and asthma. ${ }^{1,16}$ Finally, as a risk factor for recurrent wheezing developing after hospitalization, our multivariate analysis identified exposure to tobacco smoke, thus confirming the theory that indoor pollution could cause respiratory sequelae in infants. ${ }^{4}$

This study has limitations mainly because of its retrospective design. Besides, even though we studied 14 respiratory viruses, because we detected some of these viruses in few children, the small groups prevented us from reaching statistical significance.

In conclusion, our study shows that the risk factors for viral ARTIs are the presence of siblings, a family history of atopy, passive smoke and less frequent breastfeeding and the risk factor for recurrent wheezing is smoking. We also confirm as the most frequently detected viruses, RSV in bronchiolitis, hRV in pneumonia and $\mathrm{hBoV}$ in wheezing. Coinfections, mostly caused by RSV, appear not to cause more severe disease than single infections.
Along with improved breastfeeding, preventive hygiene measures intended to avoid second hand and third hand exposure to tobacco smoke might restrict viral spread from sick parents and siblings to younger children, prevent severe respiratory diseases in young children, and possibly limit sequelae.

\section{REFERENCES}

1. Kwon JM, Shim JW, Kim DS, et al. Prevalence of respiratory viral infection in children hospitalized for acute lower respiratory tract diseases, and association of rhinovirus and influenza virus with asthma exacerbations. Korean J Pediatr. 2014;57:29-34.

2. Zuccotti G, Dilillo D, Zappa A, et al. Epidemiological and clinical features of respiratory viral infections in hospitalized children during the circulation of influenza A (H1N1) 2009. Influenza Other Respir Viruses. 2011;5:528-534

3. Ruuskanen O, Lahti E, Jennings LC, et al. Viral pneumonia. Lancet 2011;377:1264-1275

4. Midulla F, Scagnolari C, Bonci E, et al. Respiratory syncytial virus, human bocavirus and rhinovirus bronchiolitis in infants. Arch Dis Child. 2010;95:35-41.

5. Franz A, Adams O, Willems R, et al. Correlation of viral load of respiratory pathogens and co-infections with disease severity in children hospitalized for lower respiratory tract infection. J Clin Virol. 2010;48:239-245.

6. Kouni S, Karakitsos P, Chranioti A, et al. Evaluation of viral co-infections in hospitalized and non-hospitalized children with respiratory infections using microarrays. Clin Microbiol Infect. 2013;19:772-777.

7. El Kholy AA, Mostafa NA, Ali AA, et al. Risk factors of prolonged hospital stay in children with viral severe acute respiratory infections. $J$ Infect Dev Ctries. 2014;8:1285-1293.

8. Fabbiani M, Terrosi C, Martorelli B, et al. Epidemiological and clinical study of viral respiratory tract infections in children from Italy. J Med Virol. 2009;81:750-756

9. Shek LP, Lee BW. Epidemiology and seasonality of respiratory tract virus infections in the tropics. Paediatr Respir Rev. 2003;4:105-111.

10. Pierangeli A, Gentile M, Di Marco P, et al. Detection and typing by molecular techniques of respiratory viruses in children hospitalized for acute respiratory infection in Rome, Italy. J Med Virol. 2007;79:463-468.

11. He Y, Lin GY, Wang Q, et al. A 3-year prospective study of the epidemiology of acute respiratory viral infections in hospitalized children in Shenzhen, China. Influenza Other Respir Viruses. 2014;8:443-457.

12. Barbosa Ramirez J, Pulido Dominguez P, Rey Benito G, et al. Human res piratory syncytial virus and metapneumovirus in patients with acute respiratory infection in Colombia, 2000-2011. Rev Panam Salud Publica. 2014;36:101-109

13. Jartti T, Lehtinen P, Vuorinen T, et al. Bronchiolitis: age and previous wheezing episodes are linked to viral etiology and atopic characteristics. Pediatr Infect Dis J. 2009;28:311-317.

14. Cilla G, Oñate E, Perez-Yarza EG, et al. Viruses in community-acquired pneumonia in children aged less than 3 years old: High rate of viral coinfection. J Med Virol. 2008;80:1843-1849.

15. Fry AM, Lu X, Chittaganpitch M, et al. Human bocavirus: a novel parvovirus epidemiologically associated with pneumonia requiring hospitalization in Thailand. J Infect Dis. 2007;195:1038-1045

16. Midulla F, Nicolai A, Ferrara M, et al. Recurrent wheezing 36 months after bronchiolitis is associated with rhinovirus infections and blood eosinophilia. Acta Paediatr. 2014;103:1094-1099.

17. Adams O, Weis J, Jasinska K, et al. Comparison of human metapneumovirus, respiratory syncytial virus and Rhinovirus respiratory tract infections in young children admitted to hospital. J Med Virol. 2015;87:275-280.

18. Wu JJ, Jin Y, Lin N, et al. Detection of human bocavirus in children with acute respiratory tract infections in Lanzhou and Nanjing, China. Biomed Environ Sci. 2014;27:841-848.

19. Martin ET, Kuypers J, Heugel J, et al. Clinical disease and viral load in children infected with respiratory syncytial virus or human metapneumovirus. Diagn Microbiol Infect Dis. 2008;62:382-388.

20. Wolf DG, Greenberg D, Kalkstein D, et al. Comparison of human metapneumovirus, respiratory syncytial virus and influenza A virus lower respiratory tract infections in hospitalized young children. Pediatr Infect Dis J. 2006;25:320-324.

21. Calvo C, García-García ML, Pozo F, et al. Clinical characteristics of human bocavirus infections compared with other respiratory viruses in Spanish children. Pediatr Infect Dis J. 2008;27:677-680. 